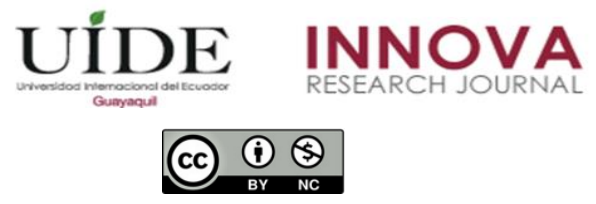

INNOVA Research Journal, ISSN 2477-9024

(Septiembre-Diciembre 2020). Vol. 5, No.3.1 pp.230-243

DOI: https://doi.org/10.33890/innova.v5.n3.1.2020.1569

URL: http://revistas.uide.edu.ec/index.php/innova/index

Correo: innova@uide.edu.ec

\title{
El Agave Andino como diversificador de la oferta turística en el Distrito Metropolitano de Quito
}

\section{The Andean Agave as a diversifier of the tourist offer in the Metropolitan District of Quito}

\author{
Víctor Elías Llugsha Guijarro \\ https://orcid.org/0000/0002-6894-9525 \\ María Soledad Oviedo Costales \\ (D) https://orcid.org/0000-0002-8080-736X \\ Walter Omar Ocaña Zambrano \\ (D) https://orcid.org/0000/0002-0266-253X \\ Universidad UTE, Ecuador
}

Autor para correspondencia: vikxox@gmail.com; sole_oviedo@hotmail.com;

walter.ocanaz@gmail.com

Fecha de recepción: 23 de septiembre de 2020 - Fecha de aceptación: 20 de noviembre de 2020

\section{Resumen}

Las actividades turísticas en el Valle Equinoccial del Distrito Metropolitano de Quito reciben a la mayor cantidad de turistas que llegan a la ciudad, esto se debe en gran medida a su ubicación: La Mitad del Mundo. Diversificar su oferta turística permitirá responder a las necesidades de un mercado turístico que requiere experiencias vinculadas a un turismo sostenible y que involucre a los actores locales en el proceso. La investigación establece la relación entre estos aspectos y el incipiente turismo gastronómico que existe en el Valle Equinoccial alrededor del agave andino. Las tendencias vinculadas a una mejor alimentación y la búsqueda del fortalecimiento de una identidad local que haga frente a la globalización han encontrado un nicho en el agave andino como un recurso turístico de gran potencial para Quito. Debido a las particularidades del fenómeno a estudiar, la presente investigación es exploratoria, utiliza herramientas propias de la metodología cualitativa, lo que permite identificar las particularidades del territorio y proponer un perfil de turista gastronómico que responda los aspectos planteados alrededor del agave.

Palabras claves: turismo, agave, turismo gastronómico, tendencias turísticas.

\begin{abstract}
Tourist activities in the Equinoctial Valley of the Metropolitan District of Quito receive the largest number of tourists who come to the city, this is largely due to its location: half the world. Diversifying your tourism offer will allow you to respond to the needs of a tourism market that requires experiences related to sustainable tourism and that involves local actors in the process. The research establishes the relationship between these aspects and the incipient gastronomic tourism that exists in the Equinoctial Valley around the Andean agave. The trends linked to a better diet and the search to strengthen a local identity that can face globalization have found a niche in
\end{abstract}


the Andean agave as a tourist resource with great potential for Quito. Due to the particularities of the phenomenon to be studied, this research is exploratory, it uses tools of the qualitative methodology, which allows identifying the particularities of the territory and proposing a gastronomic tourist profile that responds to the issues raised around the agave.

Keywords: tourism, agave, gastronomic tourism, tourist trends.

\section{Introducción}

El turismo gastronómico en el Ecuador es un tipo de turismo que se encuentra en proceso de crecimiento. Si bien la oferta gastronómica en el país es diversa y mantiene una relación próxima con los productores locales, existen productos que progresivamente se han separado del bagaje ancestral y espiritual en el que se construyeron. El agave y sus derivados se encuentran presentes en la cultura andina del Ecuador, en el Valle Equinoccial del Distrito Metropolitano de Quito, se encuentran varios espacios que de forma aislada mantienen la producción de distintos derivados del agave andino («El agave», 2019).

En el caso de Ecuador, el agave es percibido como una planta que está asociada al mundo de lo rural, del campo; es utilizada en su mayoría como una frontera viva para realizar separación de terrenos a manera de linderos, también como alimento para los cerdos. Sin embargo, dos aspectos han permitido que progresivamente se dé un cambio sobre esta percepción: a) las tendencias vinculadas al comer sano, b) búsqueda de una identidad local.

En tal sentido, la potencialidad turística del producto ha ganado espacio y ha sumado actores. En el caso del Distrito Metropolitano de Quito, existen varios emprendimientos vinculados a esta planta. En el caso del agave del Valle Occidental de Quito, su ubicación le permite contar con las condiciones necesarias para vincular a la plata y sus derivados dentro de las actividades turísticas de la ciudad, debido al hecho de que más del $80 \%$ de los turistas que llegan a Quito, visitan este sector, situación que también pone de manifiesto la necesidad de diversificar la oferta, por lo que el turismo gastronómico representa una opción de innovación para el desarrollo del turismo gastronómico a nivel local.

\section{Marco teórico}

El turismo gastronómico es relativamente nuevo, en 1998 se empieza a hablar de un turismo culinario para definir la idea de experimentar otras culturas a través de la comida (Kivela y Crotts, 2016); a decir de Long (1998), el turismo culinario aporta valor a la experiencia final del turista en su viaje.

Se puede definir al turismo gastronómico como una experiencia de viaje hacia una región gastronómica que incluya la visita a productores de alimentos, festivales gastronómicos, ferias, mercados, degustaciones o cualquier actividad turística que guarde algún tipo de relación con el turismo. Este tipo de viaje busca experimentar y aprender de las culturas locales, por medio del conocimiento de las particularidades culinarias de la región (Hall y Sharples, 2003). 
Debido a la amplitud de ámbitos que acoge el turismo gastronómico, la Organización Mundial del Turismo (2012) señala que se requiere una definición más flexible, que se adapte a las dinámicas del turismo y sus motivaciones; en tal sentido, la definición de turismo gastronómico también acoge a turistas y visitantes que planifican su viaje total o parcialmente con el propósito de degustar la gastronomía local o realizar actividades vinculadas con la gastronomía.

\section{Figura 1}

Importancia de un interés especial por la comida como motivación para viajar.

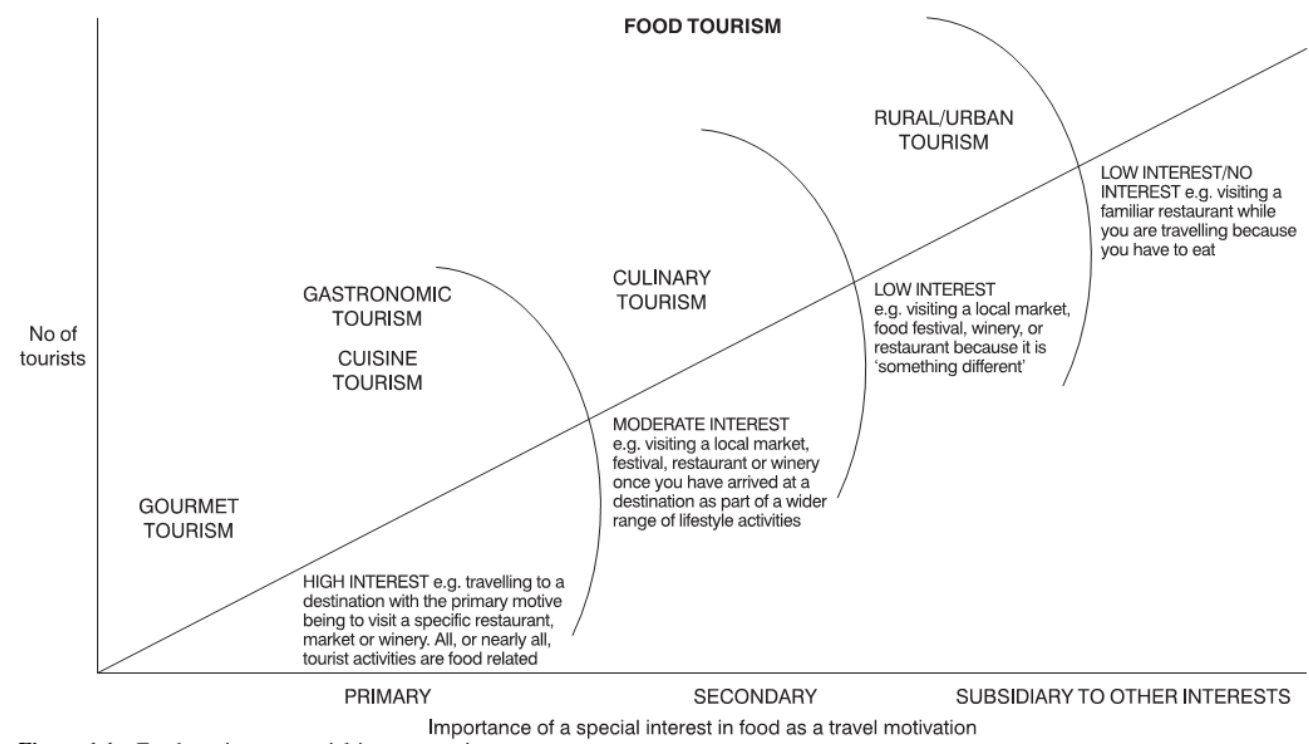

Tomado de: Hall \& Sharples, 2003, p. 11.

Más de un tercio de los gastos que realizan los turistas los destinan a la alimentación, convirtiéndose en un aspecto de gran relevancia para la experiencia de viaje, en medio de lo cual se va desarrollando de forma emergente el turismo gastronómico (World Tourism Organization (UNWTO), 2012). Sin embargo, a pesar de que es un tipo de turismo versátil, que crece de forma rápida en el mercado, las investigaciones sobre turismo gastronómico son escasas, se encuentran centradas principalmente en el vino y sus productos (World Tourism Organization (UNWTO), 2012)

La gastronomía es considerada una de las mejores vías para conocer las particularidades culturales de una población, debido a que condensa una gran variedad de actores, productos, técnicas, instrumentos, que responden a la realidad de un territorio; debido a lo cual se busca que el turismo gastronómico cuente con un discurso ético que apoye valores sostenibles, situación que se aliena a las tendencias actuales de consumo cultural. (World Tourism Organization (UNWTO), 2012). 
Los turistas que viajan motivados por la gastronomía, buscan experiencias auténticas de la localidad; el turismo gastronómico le permite consumir alimentos y bebidas, o decidir qué y dónde consumir (World Tourism Organization (UNWTO), 2017).

\section{Nuevas tendencias de consumo en el turismo}

El mundo actual se enfrenta a imparables avances tecnológicos, cambios demográficos de consumo, incertidumbre económica y nuevas preferencias de los consumidores, la reinvención de las marcas debe coincidir con las expectativas de las personas, su realidad y forma de vivir (World Tourism Organization (UNWTO), 2012).

Los nuevos consumidores buscan que sus consumos estén más allá de la compra como tal, demandan que las empresas estén interesadas por sus particularidades. La experiencia del consumo la contemplan en el sentido del consumo con: significado, sostenibilidad y transparencia (Zorraquino, 2019).

Las nuevas tendencias se generan debido a la presencia de activadores sociales que actúan impulsados por el desarrollo tecnológico y la creciente influencia de los movimientos sociales, estos actúan como una suerte de dispositivo en el cambio de paradigma del mercado y los consumidores (Zorraquino, 2019).

El turismo gastronómico como una tendencia del turismo, se encuentra relacionado con este tipo de activadores, varios de los cuales lo hacen de forma directa como la búsqueda de un estilo de vida saludable o el terreno que va ganando la gastronomía como parte de un consumo sostenible; sin embargo, como se muestra en la Tabla 1., la versatilidad del turismo gastronómico le permite interactuar con los distintos tipos de activadores sociales propuestos:

\section{Tabla 1}

Activaciones sociales y su relación con el turismo gastronómico

Activador social

Sostenibilidad

Conciencia social

Turistificación

\section{Características}

Asegurar las necesidades del presente sin comprometer las necesidades de futuras generaciones es uno de los mayores desafíos a lo que se enfrenta la humanidad.

La esperanza de una sociedad sostenible está respaldada por prácticas conscientes basadas en una economía circular en la que compartir recursos resulta imprescindible

El fenómeno de la masificación turística impacta en el tejido social urbano desvirtuando los espacios y gentrificando las ciudades en la era de la globalización.
Turismo

gastronómico

Relación directa

Relación indirecta

Relación directa 


\begin{tabular}{|c|c|c|}
\hline Activador social & Características & $\begin{array}{l}\text { Turismo } \\
\text { gastronómico }\end{array}$ \\
\hline Inclusión & $\begin{array}{l}\text { El conocimiento compartido gracias a la } \\
\text { conectividad impulsa el empoderamiento } \\
\text { social a través de una conciencia colectiva } \\
\text { que alimenta la diversidad. }\end{array}$ & Relación indirecta \\
\hline Health style & $\begin{array}{l}\text { Los nuevos estilos de vida promueven un } \\
\text { desarrollo armónico del ser humano con el } \\
\text { objetivo de elevar su calidad de vida } \\
\text { mediante el seguimiento de su bienestar. }\end{array}$ & Relación directa \\
\hline Food culture & $\begin{array}{l}\text { El papel social que desempeña la } \\
\text { gastronomía es un factor clave de estilo de } \\
\text { vida en una sociedad que reivindica un } \\
\text { consumo saludable y sostenible. }\end{array}$ & Relación directa \\
\hline Ultraconexión & $\begin{array}{l}\text { Los productores digitales compiten por } \\
\text { conseguir la atención humana en un } \\
\text { mundo saturado por la cultura de la red, en } \\
\text { el que la desconexión no está al alcance de } \\
\text { cualquiera. }\end{array}$ & Relación indirecta \\
\hline Inteligencia tecnológica & $\begin{array}{l}\text { La inteligencia artificial dota a las } \\
\text { máquinas de tecnología emocional capaz } \\
\text { de reconocer las sensaciones humanas, } \\
\text { mientras continúa el debate por la } \\
\text { privacidad y la seguridad. }\end{array}$ & Relación indirecta \\
\hline
\end{tabular}

Fuente: modificado de Zorraquino, 2019.

Estos activadores sociales determinan la presencia de diez tendencias de consumo que a decir de Zorraquino (2019) determinan el rumbo del mercado:

1. Silver economy

2. No money

3. Superalimentos

4. Cultura streaming

5. Turismo consciente

6. Re-commerce

7. Bienestar digital

8. AI First

9. Brand Ideology

10. Tecnohumans

Por sus características, el turismo gastronómico se encuentra inmerso en la tendencia del turismo consciente, que busca empoderar a los actores locales en los distintos niveles de las 
actividades turísticas, con el propósito de disminuir los efectos generados por la turistificación (Llugsha, 2019), como es el caso de la expulsión de los pobladores de una localidad por la gentrificación del sitio (Oviedo, 2014).

En el caso de la tendencia de los super alimentos, se busca que los productos ultra procesados no estén presentes en la comida cotidiana, debido al impacto que genera la industria alimentaria en el ambiente, los hábitos de consumo se alinean a una conciencia medio ambiental. Las prácticas tradicionales de la cocina ganan espacio con el propósito de generar comida real para personas reales.

\section{El agave andino}

El nombre de agave proviene del término griego agavos que significa magnífico. A lo largo del continente toma varias denominaciones como: penco, maguey, cabuya o chaguar; esta planta fue domesticada en Mesoamérica hace aproximadamente 10.000 años llegando a expandirse su uso en todo el continente (Pardo, 2005).

Durante la conquista española de América fueron varios los personajes que elogiaron las características y usos del agave y sus derivados, señalando que todo lo que se necesita para vivir, comer, beber, vestir y calzar, se lo podía obtener de esta planta (Pardo, 2005). En México, la extensa tradición alrededor del agave se puede evidenciar en la popularidad con la que hasta la actualidad cuentan varios de sus productos derivados como: miel, pulque, tequila o mezcal. En la localidad de Tequila, la gran producción de bebidas espirituosas provenientes del agave llevó a la UNESCO a reconocerlo como Paisaje Cultural de la Humanidad.

Es así que a nivel global la producción del agave está asociada de manera directa con México, y en el caso de Colombia, Venezuela y Ecuador se trata de algo más bien liminar. De hecho, con la desaparición de tierras de cultivo debido al acelerado proceso de urbanización de las ciudades en la región, el conocimiento alrededor del agave va a esfumarse.

Esto afecta también al consumo de productos derivados del agave que son más populares, entre ellos su aguamiel, bebida viscosa y dulce que presenta coloración blanca y de olor herbáceo que para algunas personas resulta bastante intenso que se extrae de esta planta que en el caso de Ecuador se la conoce como chawarmishki.

Para la extracción del chawarmishki se debe esperar a que la planta madure de cinco a siete años, el tipo de suelo y el clima son factores que determinan la fluctuación en esta temporalidad. Se realiza de forma similar en distintos territorios a lo largo de la región andina en Sudamérica y México. Al identificar un agave que por su tamaño permita desarrollar el proceso, se cortan varias hojas hasta llegar al denominado corazón de la plata. En este punto se realiza un raspado en la corteza, se cubre la superficie y luego de varias horas se recoge la sabia que se acumula en el espacio raspado. Este proceso se repite a lo largo de seis meses aproximadamente, tiempo en el que de la planta se pueden extraer alrededor de seis litros de aguamiel por día, en dos o tres turnos dependiendo del sistema de extracción. 


\section{Metodología}

En términos metodológicos, la investigación es exploratoria con un enfoque cualitativo, debido a que, como se señala en el marco teórico los estudios relacionados con el turismo gastronómico son insipientes. Se realizó una revisión literaria de artículos científicos, libros y publicaciones oficiales que permitan plantear un marco de referencia del fenómeno a analizar. La información recogida fue contrastada con observación participante en uno de los sitios identificados durante la investigación; la selección del lugar se dio con base al tipo de oferta de turismo gastronómico del lugar. También se realizaron dos entrevistas a un informante clave, que lleva 15 años trabajando el agave como un producto para el turismo en el Valle Equinoccial de Quito.

\section{Resultados}

El Valle Equinoccial alberga a las parroquias de Pomasqui y San Antonio de Pichincha, el desarrollo turístico de este territorio lo posiciona como el más visitado en el Distrito Metropolitano de Quito, esto se debe en gran medida a que la presencia de un atractivo turístico jerarquía IV: La Mitad del Mundo.

\section{Figura 2}

Sitios más visitados en el DMQ

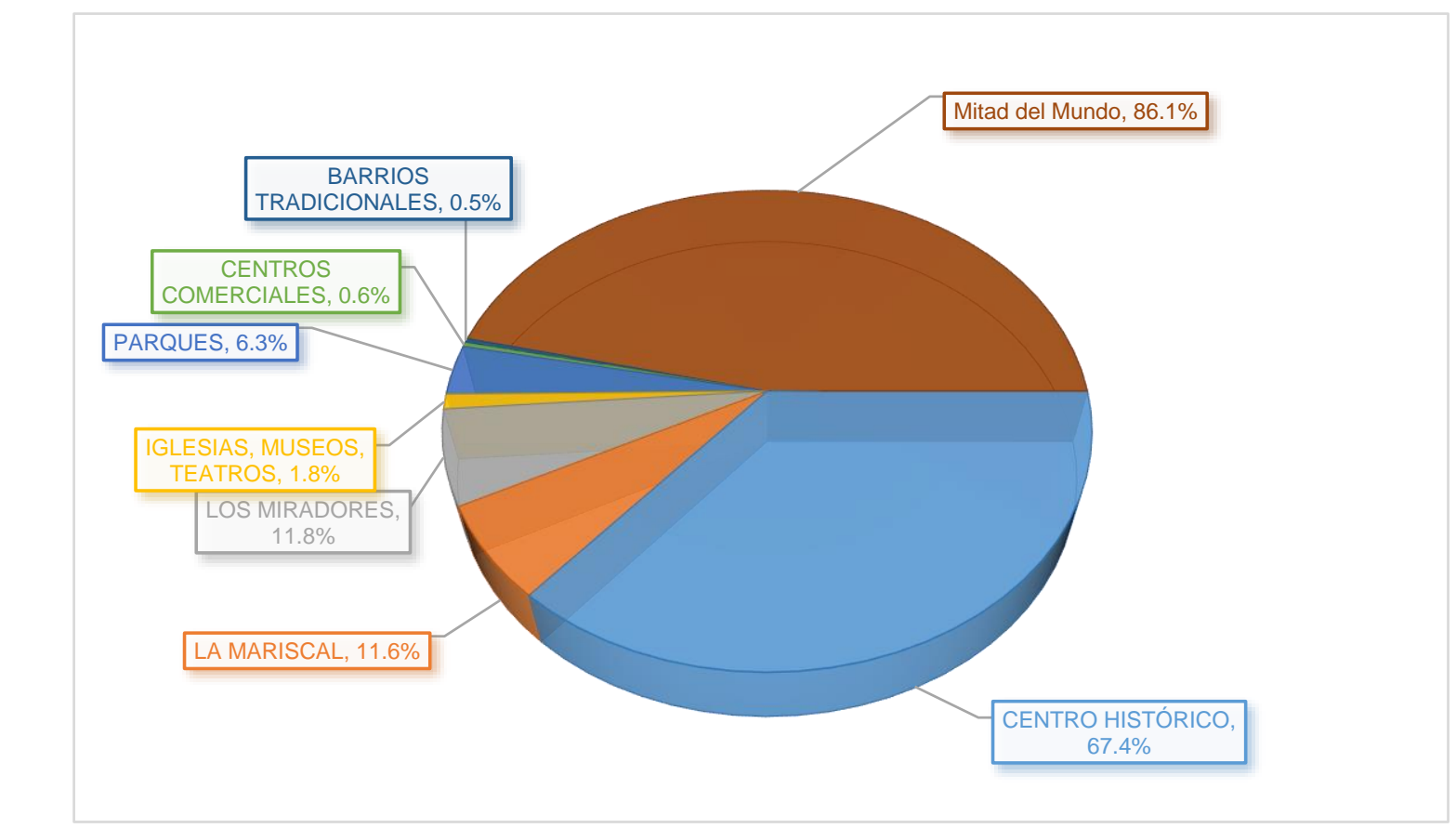

Tomado de: Quito Turismo, 2020 
La oferta turística se encuentra densificada alrededor de la Ciudad Mitad del Mundo, motivo por el cual se han propuesto opciones como la creación de rutas turísticas (Encalada, 2017) que incluyan en sus recorridos la visita de atractivos turísticos de jerarquía I y II.

Sin embargo, el único espacio que cuenta con un producto turístico consolidado en el territorio es la Ciudad Mitad del Mundo, los demás puntos son de interés local, forman parte de una insipiente oferta turística que no ha llegado a desarrollarse plenamente. La visita a la mayoría de los sitios mencionados está fuertemente ligada a la imagen que se ha construido sobre este territorio.

\section{Figura 3}

Parroquias más visitadas alrededor del DMQ

Fuente:

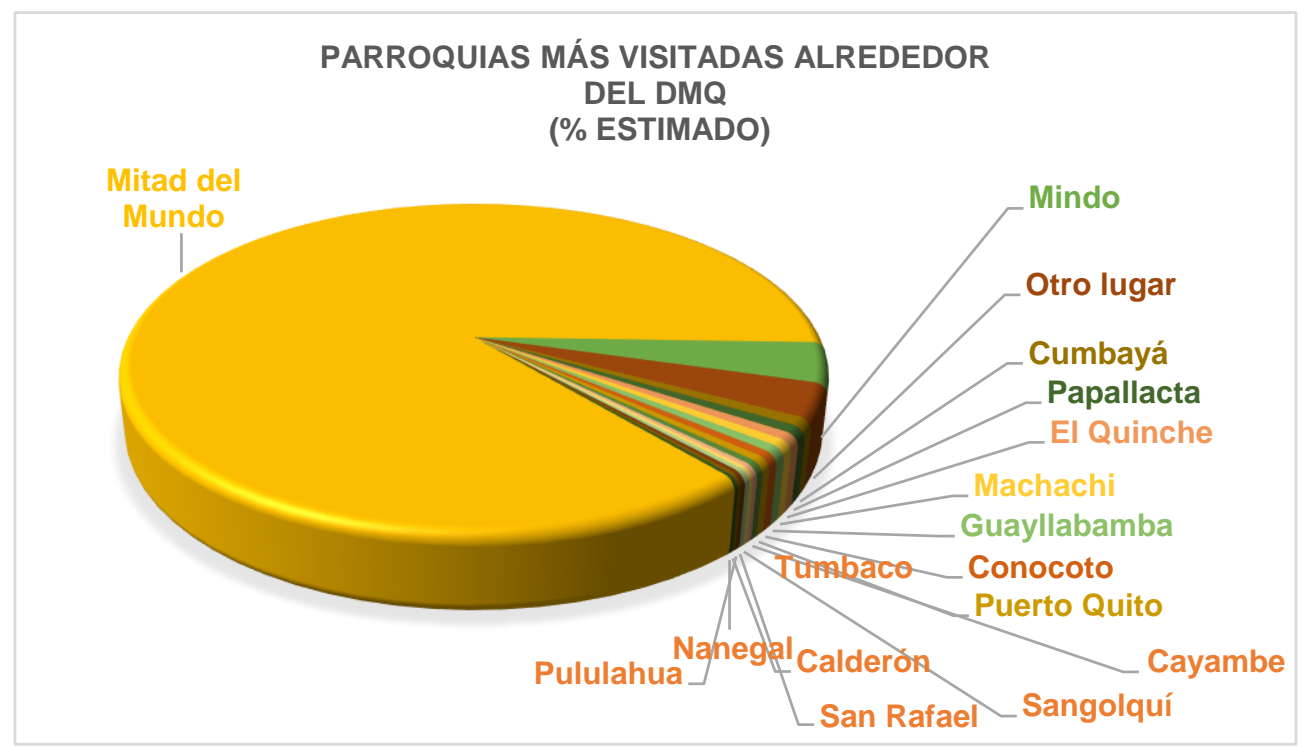

Turismo, 2020.

Quito

La oferta gastronómica que se encuentra en el Valle Equinoccial responde a una lógica que podemos denominar como cocina tradicional (Pazos, 2008), sin embargo, no llega a constituirse en una identidad vernácula de Pomasqui y San Antonio, sino que responde a una lógica regional andina.

Según datos de Quito Turismo (2020), el 31\% de turistas nacionales que llegan a la capital tienen como principal motivación la realización de actividades turísticas culturales. La motivación por la gastronomía representa el $6.1 \%$ del total. La comunicación directa es el principal medio por el cual buscan información y recomendaciones para visitar sitios y realizar actividades, especialmente sugerencias de familiares y amigos. El 72\% tiene una estadía de uno a tres días, aunque no se identifica una marcada estacionalidad, los visitantes prefieren los fines de semana para realizar el viaje, siendo la alimentación su principal gasto durante el viaje. 


\section{Figura 4}

\section{Distribución del gasto del turista del DMQ}

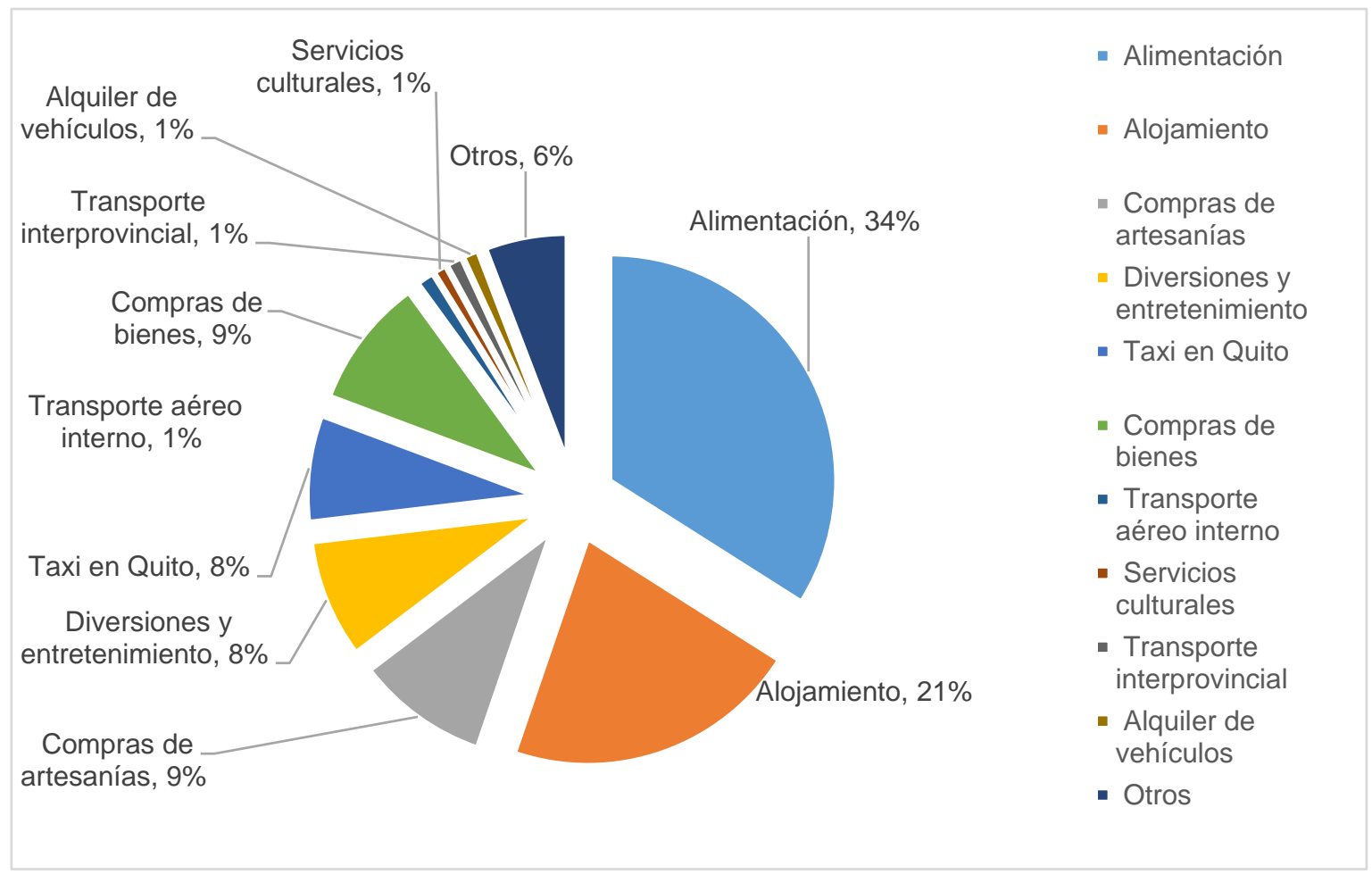

Fuente: Quito Turismo, 2020.

\section{El agave del valle occidental de Quito como recurso turístico}

En Ecuador no existe una normativa específica que regule o establezca una condición particular sobre el agave. De hecho, si bien uno de sus posibles usos es ornamental, en el Municipio de Quito (a diferencia de otras plantas), no se cuenta con especies para reforestación ni adorno en la ciudad, tampoco consta dentro del inventario de plantas ornamentales de la ciudad (D. Mora, comunicación personal, 16 de septiembre de 2020).

Por sus características, la planta del agave en general es apetecida como un alimento nutritivo. La viscosidad propia del chawarmishki generalmente aleja a los nuevos comensales, sin embargo, este suele ser utilizado como insumo para preparar varios tipos de granos y sopas.

La concentración de vitaminas y aminoácidos esenciales le hacen ser bastante particular:

100 gr. contienen 5,30 gr. de extracto no nitrogenado y $0,4 \%$ de proteínas, cantidad esta última que aunque parece baja, es interesante por su composición en aminoácidos esenciales como: lisina, triptófano, histidina, fenilalanina, leucina, tirosina, metionina, valina y arginina. Contiene vitaminas del complejo B, niacina $(0,4$ a $0,5 \mathrm{mg})$, tiamina y riboflavina, y entre 7 y 11 $\mathrm{mg}$. de vitamina $\mathrm{C}$ (el jugo de naranja fresco contiene entre 15 y $55 \mathrm{mg}$. por 100 gr.), además de hierro, calcio y fósforo. (Pardo, 2005). 
El uso alimenticio del chawarmishki del agave se encuentra presente hasta la actualidad en varias zonas de Colombia, Venezuela y Ecuador. Provincias como Imbabura, Pichincha, Cotopaxi, Loja y Azuay mantienen esta tradición, pero su consumo es anterior a la llegada de los españoles al continente americano:

Según los informantes, de ella se obtiene la llamada "miel de México" en Piura (Perú) o la llamada también miel, mermelada o chancaca de "magué" en el valle del Mantaro. El aguamiel es consumido al nivel familiar en Ayacucho (Perú), como bebida caliente puede constituir el desayuno. Fermentada, permite obtener una bebida similar al pulque conocida como chicha de "magué" (Pardo, 2005).

\section{Potencialidad patrimonial y turística}

En el caso de Ecuador, el agave es percibido como una planta que está asociada al mundo de lo rural, del campo; es utilizada en su mayoría como una frontera viva para realizar separación de terrenos a manera de linderos, también como alimento para los cerdos.

Sin embargo, dos aspectos han permitido que progresivamente se dé un cambio sobre esta percepción:

- $\quad$ Las tendencias vinculadas al comer sano y llevar una vida saludable: en los últimos años se han publicado artículos científicos que sustentan la percepción respecto al consumo del agave y su relación con la salud. Principalmente la presencia de probióticos que mejoran el sistema digestivo.

- $\quad$ Búsqueda de una identidad local: el acelerado crecimiento de la globalización y los procesos de aculturización, han llevado a distintos actores locales a realizar procesos de fortalecimiento del patrimonio local. En este marco, distintos colectivos han identificado en el agave un aspecto que debe ser puesto en valor por su histórica tradición.

En tal sentido, la potencialidad turística del producto a ganado espacio y ha sumado actores. En el caso del Distrito Metropolitano de Quito, existen varios emprendimientos vinculados al agave, sin embargo, se identificaron dos emprendimientos que realizan sus actividades alrededor del agave con mayor posicionamiento en el territorio. Los sitios fueron seleccionados considerando las actividades que realizan y en qué medida transmiten aspectos vivenciales y la vinculación del producto a la realidad del territorio.

- $\quad$ Chucchurilos Naupa Ayllu Manta: es un emprendimiento de la familia Sigcha Hidalgo, que se autodefine como conservadora y preservadora del conocimiento vinculado al agave. En este sitio se cuenta con una explicación en primera persona respecto al agave como elemento cultural gastronómico para las familias de la localidad. En la visita se puede acompañar a un miembro de la familia a realizar el proceso de extracción del miske del agave.

Adicionalmente entre los productos que venden se encuentran otros derivados del agave, entre los que se destacan esculturas y alpargatas. 
- $\quad$ Casa Agave: la conceptualización de este emprendimiento se enmarca en los aspectos espirituales del agave, su vinculación con la tierra, la alimentación y el medio ambiente. Cuentan con un espacio diseñado a manera de museo donde los visitantes realizan actividades interactivas asociadas al agave. En su oferta cuentan con un ejercicio de cata de bebidas espirituosas derivadas del agave. Entre los productos adicionales que ofertan se encuentran: jugos, cerveza y licor a base miske. (Agave Spirit, s. f.).

Casa Agave es el sitio que ha logrado posicionarse dentro del circuito turístico de la ciudad de Quito. Este emprendimiento hace énfasis en el agave partiendo desde una perspectiva cultural, señalando al chawarmishki como la "bebida de la eterna juventud y el regalo de los dioses"(Agave Spirit, s. f.).

Su ubicación le permite contar con las condiciones necesarias para vincular sus productos dentro al turismo. En una serie de acciones progresivas, han pasado de tener un jardín, un museo, un espacio de degustación («El agave», 2019), a contar con la oferta de un full day de actividades vinculadas al agave en el sector de la Mitad del Mundo a propósito de una alianza estratégica con Quito Tour Bus, lo que permitió que para finales del 2019 el $85 \%$ de los pasajeros que visitaban el sitio sean extranjeros (D. Mora, comunicación personal, 16 de septiembre de 2020).

La propuesta de Casa Agave se alinea a los activadores sociales que generan tendencias en el consumidor:

- $\quad$ Sostenibilidad: como parte del recorrido los visitantes siembran una planta de agave, la cual es depositada en un pequeño vivero, para posteriormente ser trasplantada en el cerro del Casitagua. De esta forma realizan un proceso de reforestación con una planta nativa del sector.

- $\quad$ Health style: los beneficios de la inclusión de los productos del agave en la dieta diaria, se alinea con el objetivo de mejorar la calidad de vida de las personas.

- $\quad$ Food culture: durante el recorrido se visita el Museo del Agave, donde se pone de manifiesto el rol que juega esta planta en la vida de los habitantes del Valle Equinoccial que mantienen la tradición de su uso.

- $\quad$ Conciencia social: el chawarmishki que utilizan para realizar sus productos proviene de 8 familias del sector, situación que ha permitido el empoderamiento de mujeres cabeza de hogar en torno a la economía circular. (D. Mora, comunicación personal, 16 de septiembre de 2020).

A estos factores se deben sumar tres elementos que resultan fundamentales en el mercado turístico: calidad, autenticidad, experiencial. En lo que respecta a la calidad, es importante considerar que no toda propuesta es válida, esta debe responder a los estándares del mercado en el que se quiere posicionar (Cantú, 2011). Sobre la autenticidad, esta es percibida por los turistas no como la construcción de un discurso, si no como la posibilidad de evidenciar la permanencia de la manifestación entre habitantes de la localidad. Finalmente, lo experiencial responde a la necesidad del mercado de sentirse parte de las actividades en el territorio, abandonado el rol de mero espectador (Hernández et al., 2019). 


\section{Perfil del turista gastronómico}

El turista gastronómico en el Ecuador en su mayoría lo es por accidente o como una consecuencia del desarrollo de actividades turísticas que desarrollan en el destino. En tal sentido este tipo de turista no realiza su viaje de forma exclusiva por temas gastronómicos, si no que los identificacomo importantes en el desarrollo de las actividades de su visita, haciendo énfasis en el hecho de que probar platos tradicionales los vincula a la necesidad de valor la gastronomía como parte del patrimonio local.

Debido a que, de forma general, el perfil de turista que visita Quito es de una persona con estudios superiores (61\%), su edad fluctúa entre los 18 y 45 años y tomando en cuenta el proceso de puesta en valor de los conocimientos ancestrales vinculados a la planta del agave y sus usos, el denominado Turista Cosmopolita es quien encaja de mejor manera en la propuesta.

Los turistas que llegan a Quito en su mayoría no utilizan canales convencionales para organizar su viaje (agencias de viaje), el 37\% se entera del destino por medio de internet y un $41 \%$ por medio de familiares y amigos, se plantean las siguientes acciones de promoción y comercialización.

\section{Tabla 2}

Propuesta de comercialización y promoción del agave para un perfil de turista cosmopolita

\begin{tabular}{l|l}
\hline Perfil de turista & Acciones de comercialización y promoción \\
\hline \multirow{4}{*}{ Turista cosmopolita } & $\begin{array}{l}\text { Creación de cuentas oficiales de la ruta en: Facebook e } \\
\text { Instragram donde se brinde información relevante sobre la } \\
\text { ruta. }\end{array}$ \\
\cline { 2 - 2 } $\begin{array}{l}\text { Establecer alianzas con las instituciones públicas; Quito } \\
\text { Turismo y Ministerio de Turismo, para que se incluya la ruta } \\
\text { en las guías oficiales de viaje. }\end{array}$ \\
\cline { 2 - 2 } $\begin{array}{l}\text { Publirreportajes en medios de comunicación masiva, donde } \\
\text { se destaque el proceso de puesta en valor de la ruta. }\end{array}$ \\
\cline { 2 - 2 } \\
$\begin{array}{l}\text { Famtrips con foodies que se identifiquen con la valoración } \\
\text { del patrimonio local. }\end{array}$ \\
\hline
\end{tabular}

Fuente: elaboración propia.

Debido a lo incipiente de la oferta local y que las certificaciones de calidad y denominación de origen son aspectos que aún se encuentran en construcción, se busca asociar a este tipo de turista con dos niveles de oferta: a) degustación, b) cata.

La degustación se puede vincular a un proceso en el que el turista recibe una explicación del producto y realiza un recorrido por plantaciones de agave donde observa el proceso de extracción del miske. 
La cata, estará vinculada a un proceso de explicación de los elementos ancestrales y espirituales de la planta, para luego participar de un ejercicio guiado de cata de distintos productos derivados del agave.

\section{Conclusiones}

El desarrollo del turismo gastronómico en el Valle Occidental de Quito tiene un gran potencial, debido al encadenamiento que existe con su principal atractivo turístico, sin embargo, captar este tipo de mercado requiere una serie de acciones que deben ser llevadas a cabo a nivel público y privado.

El agave y su consumo deben asociarse a un proceso de educación y puesta en valor, debido principalmente a que esta planta y sus derivados se asocian de forma general al ámbito rural e indígena como un aspecto negativo.

Se sugiere establecer un proceso que lleve a la creación de una denominación de origen del agave, que busque destacar las particularidades respecto a la plata y los aspectos organolépticos que se general según el suelo, altura y clima del Valle Equinoccial.

Debido a su versatilidad, el desarrollo del turismo gastronómico resulta una opción pertinente para la diversificación de la oferta turística del Distrito Metropolitano de Quito, se debe captar al mercado local con el propósito de empoderar a la población residente respecto a su historia y su territorio.

\section{Referencias Bibliográficas}

Agave Spirit. (s.f.). Chawarmisqui. Recuperado 10 de julio de 2020, de http://agavespiritecuador.com/?page_id=435

Cantú, J. (2011). Desarrollo de una cultura de calidad. McGraw-Hill.

El agave. (2019, agosto 26). En Día a Día. https://www.youtube.com/watch?v=WV-af9M-1y0

Encalada, E. (2017, octubre 5). La cultura equinoccial es un atractivo turístico. El Comercio. http://www.elcomercio.com/viajar/cultura-equinoccial-atractivo-turistico.html

Hall, M., \& Sharples, L. (2003). The consumption of experiences or the experience of consumption? An introduction to the tourism of taste. En The consumption of experiences or the experience of consumption? An introduction to the tourism of taste (pp. 1-24). Elsevier.

Hernández, J., Pasaco, B., \& Campón, A. (2019). Community-based tourism from the experiential approach: A way of tourist's participation with local communities. Journal of Tourism and Heritage Research, 2(4), 90-122. 
Kivela, J., \& Crotts, J. (2016). Tourism and Gastronomy: Gastronomy's Influence on How Tourists Experience a Destination: Journal of Hospitality \& Tourism Research. https://doi.org/10.1177/1096348006286797

Llugsha, V. (2019). La ciudad de las oportunidades. Turistificación y emprendimientos del Centro Histórico de Quito. INNOVA Research Journal, 4(3.1), 223-232. https://doi.org/10.33890/innova.v4.n3.1.2019.1065

Long, L. (1998). Culinary Tourism. University Press of Kentucky.

Mora, D. (2020, septiembre 16). Entrevista Casa Agave [Comunicación personal].

Oviedo, S. (2014). Centro histórico de Quito: Cambios en la configuración residencial y usos de suelo urbano asociados al turismo. [Maestría, Pontificia Universidad Católica de Chile]. https://estudiosurbanos.uc.cl/en/exalumnos/centro-historico-de-quito-cambios-en-laconfiguracion-residencial-y-usos-de-suelo-urbano-asociados-al-turismo/

Pardo, O. (2005). El agave americana (Agave Americana L.): Uso alimentario en el Perú. Chloris chilensis, 2. https://www.chlorischile.cl/agavepardo/Agavetexto.htm

Pazos, J. (2008). El sabor de la memoria: Historia de la cocina quiteña. FONSAL, Fondo del Salvamento del Patrimonio Cultural de Quito.

Quito Turismo. (2020). Quito en cifras. http://quito-turismo.gob.ec/estadisticas/

Quito Turismo, \& Universidad Central del Ecuador. (2020). Encuesta sobre el comportamiento de los residentes en Ecuador que visitan Quito. Quito Turismo.

World Tourism Organization (UNWTO) (Ed.). (2012). Global Report on Food Tourism (Vol. 4). World Tourism Organization (UNWTO). https://doi.org/10.18111/9789284414819

World Tourism Organization (UNWTO). (2017). Second Global Report on Gastronomy Tourism (Vol. 16). World Tourism Organization https://doi.org/10.18111/9789284418701

Zorraquino, $\quad$ M. $\quad$ (2019). $\quad$ Consumer $\quad$ Trends 2020. https://www.zorraquino.com/sesiones/2019/11/tendencias-consumo-2020.html 